\title{
Malaria Intermittent Preventive Treatment (IPTi) pharmacovigilance in Malawi: A case of Lilongwe district.
}

\author{
Prestor J Kubalalika1, MPH \\ 1. Director, REP-Public Health Consultants (REPPHECO), Malawi.
}

\begin{abstract}
Background: Intermittent Preventive Treatment with Sulfadoxine-Pyrimethamine in infants (SP-IPTi) is a malaria control strategy which, together with the delivery of routine childhood immunizations, as recommended by the World Health Organization (WHO) was implemented in Lilongwe district of Malawi from September 2008 to November in 2009. A study was performed by Lilongwe District Health Office (DHO) in collaboration with funding from UNICEF to evaluate the safety of SP-IPTi and identify potential new Adverse Events (AEs) spontaneously identified, reported, monitored and evaluated.

Methods: A cohort event monitoring study was conducted on 15, 000 infants in 4 Health Facilities (HFs) after administration of SP-IPTi to infants during routine immunizations. A total of about 50 Community Health Workers (CHWs) and volunteers were trained in pharmacovigilance and were supervised by senior personnel in all the five HFs.

Infants received half tablets of SP immediately after receiving DPT-HepB+Hib (Pentavalent) 2 vaccine / (IPTi 1), Pentavalent 3 / (IPTi 2) at 10 and 14 weeks respectively and Measles vaccines/(IPTi 3) at 9 months. These children were recorded and their mothers were given diary cards with pictures of possible AEs. Community Health Workers (CHWs) and volunteers followed up every child after 10 days of administration/registration to collect the diary cards where parents indicated types of AEs observed on their children as well as starting and end dates of such possible AEs.

The indicated AEs were entered into a computer database from all the collected diary cards according to HFs. Possible side effects/AEs that were looked for were; persistent crying, fever, vomiting, diarrhoea, skin rashes, abdominal pains, insomnia, nausea, mouth sores, and itching among other related possible side effects.

Results: A total of 15,105 children received the IPTi and were followed in all four health facilities. Out of this, $50.3 \%(7,594)$ were male while $49.7 \%(7,511)$ were females. Of these, $19.2 \%$ [1247], $95 \% \mathrm{Cl}$ (276-304) developed AEs as follows; $42 \%$ persistent crying, 28\% fever, 18\% vomiting, $5.2 \%$ skin rashes and $6.8 \%$ presented with other minor symptoms while $80.8 \%(13,858)$ did not develop any side effect. $43.2 \%$ (1254) of those who showed symptoms were IPTi1 recipients, 35.3\% (1022) received IPTi2 while $21.5 \%$ (624) were from those who received IPTi3.

Conclusions: This study showed that simultaneous administration of SP-IPTi together with immunizations was a safe strategy for implementation with very minimal serious AEs to infants. In this case therefore, strategies towards strengthening such spontaneous reporting in Malawi should not only be left to service providers but also to beneficiaries or their caregivers.
\end{abstract}


Correspondence: prestorkubalalika@gmail.com

DOI: 10.5210/ojphi.v11i2.9956

Copyright $\odot 2019$ the author(s)

This is an Open Access article. Authors own copyright of their articles appearing in the Online Journal of Public Health Informatics. Readers may copy articles without permission of the copyright owner(s), as long as the author and OJPHI are acknowledged in the copy and the copy is used for educational, not-for-profit purposes.

\section{Literature Review}

SP-IPTi is the administration of a full therapeutic course of SP delivered through the Expanded Programme on Immunization (EPI) at intervals corresponding to routine vaccination schedule for the second and third dose of DPT/Penta and measles vaccination-usually at 8-10 weeks and 9 months of age-to infants at risk of malaria (WHO 2011).

Pharmacovigilance is defined as the science and activities relating to the detection, assessment, understanding and prevention of adverse effects or any other drug-related problem. The aims of $\mathrm{PV}$ are to enhance patient care and patient safety in relation to the use of medicines; and to support public health programmes by providing reliable, balanced information for the effective assessment of the risk-benefit profile of medicines (WHO 2018).

Jeetu $G$ et al (2010) described Pharmacovigilance as a sunshade to describe the processes for monitoring and evaluating Adverse Drug Resistances (ADR) and it is a key component of effective drug regulation systems, clinical practice and public health programmes. This would consider litigious and important drug safety issues that have the potential to affect public health adversely beyond national boundaries.

In addition, European Medicines Agency (2016) stated that the process of pharmacovigilance involves several distinct steps such as collection of information on potential side effects, detecting if any new or changing side effects have arisen, deciding if action is needed to optimize the safe and effective use of the medicine and then communicating this to the users of the medicine.

Coomarasamy A et al (2016) said that any untold medical occurrence in a participant, which does not necessarily have a causal relationship with the trial event is called an Adverse Event and that any untold and unintended responses to the trial intervention, at any dose administered, including all AEs judged by either the reporting investigator or sponsor as having a reasonable causal relationship to the trial intervention is called Adverse Reaction. Walshe K (2000) stated that Adverse Events are instances which indicate or may indicate that a patient has received a poorquality care.

\section{Methods}

The pharmacovigilance study was performed on all under one-year-old children from the four health facilities of Kawale, Mitundu, Chiwamba and Likuni in Lilongwe district of Malawi. Infants were given half tablets of SP immediately after receiving Pentavalent 2, 3 and measles vaccines at 
6, 14 weeks and 9 months respectively. CHWs and volunteers who were trained on follow up and collection of diary cards were recording every child based on the location each child was coming from. Mothers/caregivers of these children were given diary cards with pictures of possible side effects on them. They were asked to indicate/check alongside any side effect they observed on their children and to also indicate the starting and finishing dates of observed side effects. These cards were issued immediately after children received immunization and SP right at the clinic site. Then responsible CHWs and/or volunteers from where these children were coming, took note of their location details for easy follow-up. The possible side effects indicated on the diary cards were; persistent crying, fever, vomiting, skin rashes, diarrhoea, abdominal pains, nausea, itching, insomnia and any other they would observe. After ten days, CHWs and/or volunteers were following up every child at their homes to collect the diary cards from their mothers or caregivers. The collected diary cards were then taken to the program coordinator who later entered all AEs indicated on these cards into the database.

In the event of a suspected Serious Adverse Event (SAE), immediate reports were sent to relevant supervisors who in turn made quick follow ups where thorough diagnosis was made and/or samples taken. These were then sent to paediatric experts at Kamuzu Central Hospital ( $\mathrm{KCH})$ for examination and responsible suspects (children) were assessed before making a confirmation or otherwise if the reaction was indeed due to SP or not.

\section{THE DIARY CARD}

The card was where information on symptoms was entered by parents/caregivers and it contained pictures of possible AEs. The left part of the card was where detailed information about the child was indicated for easy and proper follow up. The right part of it was where parents/caregivers were indicating any of the suspected possible symptoms they observed on their children. This was the most important part of the diary card. Parents/caregivers were supposed to check/tick in the boxes next to pictures which corresponded to symptoms children showed and then they were indicating the starting and finishing dates of such symptoms. Then there was a table on the far right of the card which contained other possible symptoms to look for and indicated accordingly. At the immediate bottom of that table was the provision for outcomes which were supposed to check if any of it happened.

This card was later followed-up after ten days from administration of IPTi and collected by CHWs and/or responsible volunteers. The card was then handed over to the study coordinator who entered the information in the computer database. These cards were finally filed together per health facility. If by any chance the coordinator suspected a Serious Adverse Event (SAE), he would use the information from the this card to make further follow ups to the children beginning with the health facility it came from where confirmations were made that either the children still had the symptoms or had since recovered before deciding to forward/transport the case to experts at the referral Kamuzu Central Hospital for further analysis and/or action otherwise they would rule the case out of possible SAE right there at HF level.

Before parents/caregivers were given this card at the immunisation clinic site, CHWs and volunteers were explaining to them on how to use it. They also advised them on the importance of the card and that they should properly keep it. They were also told that after ten days, either the $\mathrm{CHW}$ or the volunteer of their area would come to collect the card. They were further advised that 
in case they noticed and suspected any SAE within the ten days before the arrival of the $\mathrm{CHW} /$ volunteer, they shouldn't wait but rush with the child to their nearest HF for medical attention (see appendix 1).

\section{DATA ENTRY}

Information from the diary card was entered into a computer database where all doses received were indicated plus statuses of symptoms (if the cards showed any). If, however the child did not present with any symptom, the diary card was still collected from the parent/caregiver and information to the same was entered into the database. This means that on this database, only the top part (which gave details of the child's name and location of its residence) was entered leaving the symptom part since the child did not show any symptoms. Every child's record was dully kept by the coordinator such that after IPTi1/Pentavalent 2, the child was expected to come for the second time for IPTi 2/Pentavalent 3 and another card was given which was later handed over to the coordinator for entry until the third dose at 9 months when the child got measles vaccine together with SP-IPTi3 dosage. This database contained space for all the three IPTi doses and after the child had finished receiving the three doses and his/her data entered, then he/she was regarded as fully covered (see appendix 2).

\section{RESULTS}

Out of 15,105 total enrolments, 50\% (7,555) received IPTi-SP while the other 50\% $(7,550)$ did not receive. Among those who did not receive, were those who were not yet due for IPTi (those who got Penta 1 at 6 weeks) and for those who were enrolled, but for some other reasons, their mothers/caregivers refused to continue taking part plus those that were untraceable/unaccounted for due to unidentified relocations and/or those who deliberately gave false addresses. However out of 7,555 who received IPTi, 2,900 (38.4\%) reacted while 4,655 (61.6\%) did not. Therefore, in this case, out of the total 15,105 who enrolled, $19.2 \%(2,900)$ reacted and $80.8 \%(12,205)$ did not. In all, there were 7,594 (50.3\%) male and 7,511 (49.7\%) female infants. Of the total 2,900 (19.2\%) children with symptoms, 1,219 (42\%) showed persistent crying which was due to (among other reasons) the vaccine injection they were given during immunisation. $812(28 \%)$ had fever which would also be as a result of the vaccine, 524 (18\%) vomited, 160 (5.5\%) developed mild skin rashes which lasted for a few days, $82(2.8 \%)$ had diarrhoea, $42(1.4 \%)$ presented with insomnia, $18(0.6 \%)$ mild abdominal pains, $12(0.4 \%)$ had itching, $10(0.3 \%)$ dark cloured urine while 21 $(0.7 \%)$ had shown other minor symptoms.

Out of all those who had the above symptoms, no one had shown Serious Adverse Event due to SP within the 10 days monitoring period. Most of these children had mild reactions which took not more than 2 days and no one was admitted for the whole monitoring period of the study. Crying and fever were more prevalent $(98.8 \%)$ than the rest which were attributed to vaccines and some mild sores due to injections which were normal to mothers/caregivers that evidently lasted for a few days and were treated with some pain killers and tapped sponging on injected areas/sites. To those who showed diarrhoea, insomnia, abdominal pains, itching, dark cloured urine, the reactions took not more than two days such that most of them disappeared/ceased on their own without medical treatment. 
As shown in figure 2, of the three IPTi doses, IPTi 1 presented with more symptoms (43.2\%) followed by IPTi $2(35.2 \%)$ and IPTi $3(21.6 \%)$. As already explained above, the more the number of children per IPTi dose, the higher the symptom coverage. This means that IPT1 had more children who received the IPTi-SP than the other two.

Table 1: Vaccine/SP enrollment and IPTi symptoms

\begin{tabular}{|c|c|c|c|c|c|c|c|c|c|}
\hline \multirow[t]{3}{*}{ H/Facility } & \multicolumn{6}{|c|}{ Sex } & \multirow{3}{*}{$\begin{array}{c}\text { Number } \\
\text { with } \\
\text { Symptoms }\end{array}$} & \multirow{3}{*}{$\begin{array}{c}\text { Number } \\
\text { without } \\
\text { symptoms }\end{array}$} & \multirow[t]{3}{*}{ Total } \\
\hline & \multicolumn{2}{|c|}{ Male } & \multicolumn{2}{|c|}{ Female } & \multicolumn{2}{|c|}{ Total } & & & \\
\hline & Vacc & SP & Vacc & SP & Vacc & SP & & & \\
\hline Chiwamba & 1,480 & 740 & 1511 & 756 & 2,991 & 1,496 & 521 & 2470 & 2,991 \\
\hline Kawale & 2,345 & 1173 & 2,173 & 1087 & 4,518 & 2,260 & 839 & 3679 & 4,518 \\
\hline Likuni & 1,232 & 616 & 1371 & 686 & 2,603 & 1,302 & 553 & 2050 & 2,603 \\
\hline Mitundu & 2537 & 1269 & 2456 & 1228 & 4,993 & 2,497 & 987 & 4006 & 4,993 \\
\hline Total & 7,594 & 3,798 & 7,511 & 3757 & 15,105 & 7,555 & 2,900 & 12,205 & 15,105 \\
\hline
\end{tabular}

This table shows the enrollment of all 15,105 children. The sex section is split into male/female and total columns. Each column i.e. male, is again split into two i.e. "total of those who received vaccines" (sum of Penta 1,2 \& measles) which is labeled "vacc". Then the other column "SP" means that out of those who got vaccinated, how many received IPTi-SP.Below is the enrollment coverage chart for children who received SP-IPTi per dosage;

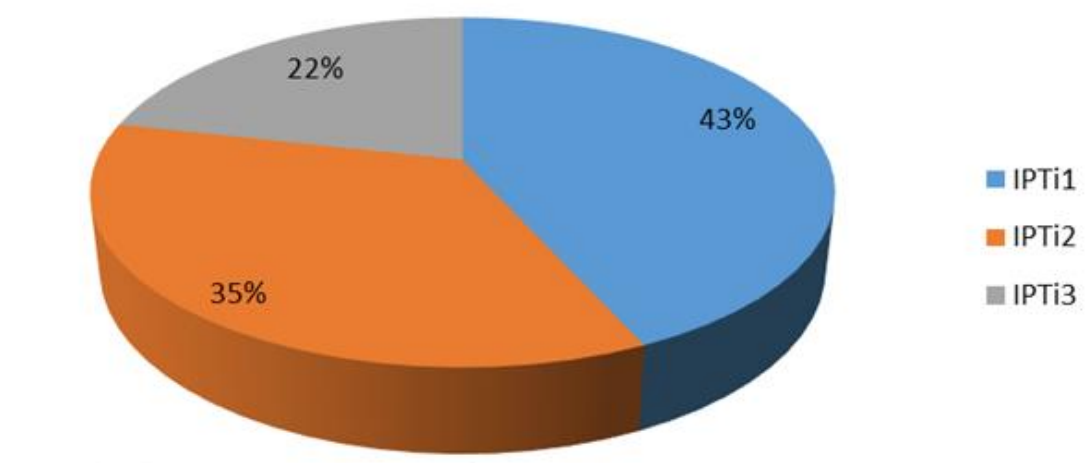

IPTi Coverage per dosage

Fig 1: Coverage of SP-IPTi per dose

The above chart shows coverage for each IPTi dosage given (1-3) which shows that there were more children that received IPTi1 at $43 \%$ i.e. $(6,495$, of the total 15,105 children who were enrolled) that was concurrently given with Pentavalent 2 vaccine (at 10 weeks) than the other two IPTi doses. The graph shows that utilization was dropping (from $43 \%$ in IPTil to $22 \%$ IPTi3) after every dose given such that by the time these children were receiving IPTi3 at 9 months during measles vaccine, $21 \%$ of those who got first dose had dropped out as has always been the case with childhood immunisation. Some of the reasons for this drop out was that there was no further 
follow-up to those who dropped-out and/or some children had received IPT1 with third Pentavalent dosage (at 14 weeks) and/or measles hence, they couldn't have come back again for the remaining IPTi dosage since they would have already finished their corresponding vaccination dosage.

The graph below shows number of children who received IPTi as above that had developed symptoms per dosages;

\section{Fig 2: IPTi symptoms per dosage}

Number of children with symptoms against doses

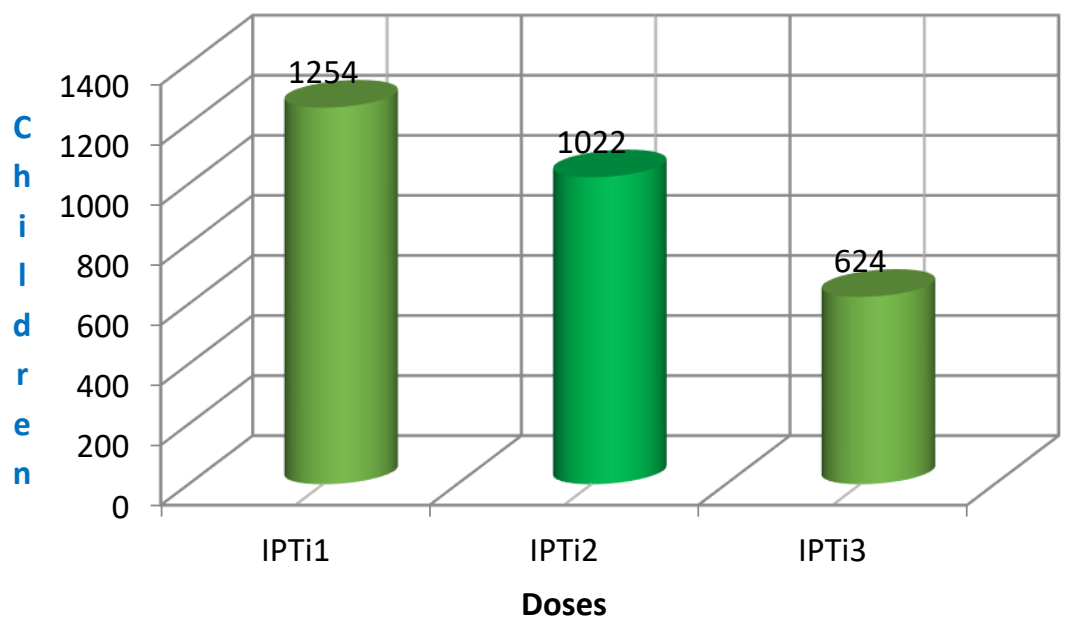

The above graph shows children who developed symptoms after taking SP-IPTi (1-3). There were overall, more children who received IPTi1 that reacted and presented with symptoms i.e. $(1,254$ out of the total 2,900 who reacted) than the other two which was also the case as the trend kept declining with number of doses given. This was so because obviously there were already more children who received IPTil than the other two as shown in the previous chart. This scenario therefore explains that; the more the children who received a particular IPTi dose, the more symptoms were shown and vice versa. Although some of those who reacted previously returned for later doses, the number of returnees was still lower than of those who came before and this was the case in all the three doses.

Below however is a graph showing children who presented with various specific symptoms; 


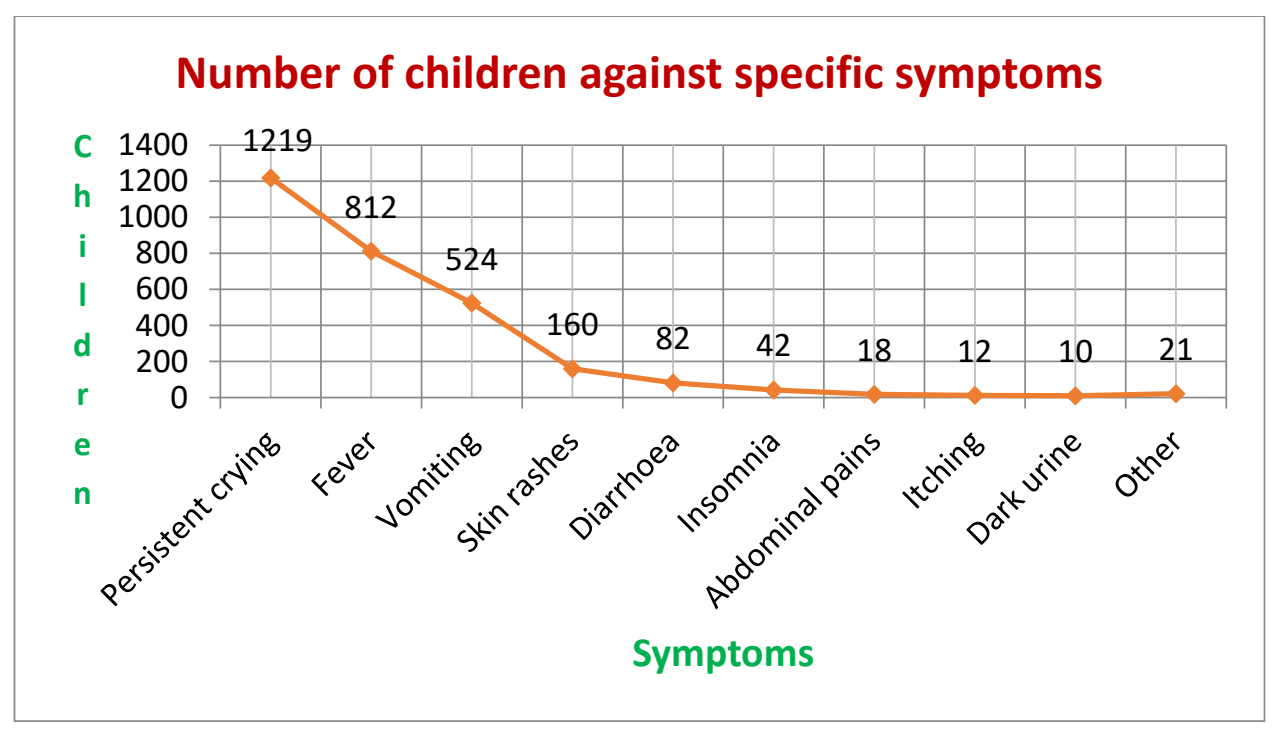

Fig 3: Specific IPTi symptoms

The graph shows that out of 2,900 children who were followed and reacted after 10 days of administration, most of them presented with persistent crying $(1,219)$ then fever $(812)$ and the least was dark cloured urine as seen in the above graph.

\section{INTERPRETATION/DISCUSSION}

The fact that $19 \%$ of 15,105 children reacted and showed symptoms after taking IPTi-SP, this coverage however, did not present any Serious Adverse Event following SP administration as would be anticipated. Most of the symptoms were mild which took a few days to subside, be healed and/or treated. There were no any hospital admissions made although some of them had been referred to experts after showing some symptoms like skin rashes but these were eventually ruled out of any danger of SAEs for they subsequently subsided within 10 days of active surveillance period. In this case therefore, it was clear that using SP as a preventive treatment to infants against malaria together with immunisation was safe and that most of the symptoms that manifested were due to some mild reactions which were not as serious to children that were promptly treated and/or managed.

The study therefore showed that SP-IPTi could effectively be used to children together with immunisation in preventing malaria in infants as was the case with IPTp in pregnant women (according to World Health Organization). The study also proved that IPTi could be rolled out to other districts of the country taking into account its safety on the health of infants during this period.

On the other hand, in all four health facilities, clinicians indicated (though anecdotally) that number of infants presenting with malaria was drastically reduced as compared to previous months. As a result of this, they were not seeing as many children as in previous months before the study and as a result, these health facilities were not running out of anti-malaria drugs as was previously the case when they were seeing and treating a lot of children against malaria (this assertion could not be scientifically proven with data evidence). 
Mothers/caregivers on the other hand, commended the initiative as their children were protected from malaria such that they were able to have more time doing household chores than frequenting health facilities to have their children treated against the disease which was so prevalent among infants. They as a result, also had enough time to do some other things in their homes for their families such as business without being interrupted by going to health facilities to have their children treated for malaria.

This also brought financial stability in some homes who more often were used to frequently buying anti-malaria treatment from shops and/or private clinics whenever their children were sick from the disease. Due to this, they would save the money they could use to buy the drug to buying some basic household needs.

To families who could not afford buying ITNs, for their children this was a huge relief as although they were exposed to mosquito bites, their children were on the other hand assured of not catching malaria due to IPTi protection. Some mothers/caregivers who were staying far away from health facilities regarded this initiative as worthwhile for they were able to save some money they would use on transport to and from health facilities for buying food and other items in their homes.

In addition to this, the study found out that it also helped increase immunisation attendances since mothers/caregivers brought their children for IPTi which in the process, helped raise the immunisation coverage of these health facilities that enabled them reach their immunisation target populations as expected. Mothers/caregivers frequented immunisation clinics to have their children administered with SP-IPTi at the same time receive vaccinations. This also helped reduce the EPI immunisation drop-out rates in these facilities hence it was seen as a service which made them kill two birds with one stone i.e. protecting their children against childhood immunisable diseases by vaccines as well as malaria by IPTi.

The IPTi pharmacovigilance therefore showed that it was possible and safe to combine the two (immunisation and $S P$ ) on infants without registering any Serious Adverse Event. It therefore proved that doing this would effectively address two problems at once which would help save children's lives against corresponding diseases. It also proved that it would reduce the burden on clinicians of seeing many children with malaria which would eventually enable them serve more other patients with different diseases hence improve the health of both infants and other patients.

\section{LIMITATIONS}

Despite the fact that the study was successfully done and that its results were encouraging, not all children were adequately followed during the study period from IPTi1 to IPTi 3 . There was $21 \%$ drop-out rate which was due to several factors and misconceptions/myths as well as suspicions against the study being one of them. Some women/caregivers dropped out due to poverty which made them fail to finish attending clinics for lack of transport money to those living far away from immunisation clinic sites as well as good clothes to put on during such clinics (although it wasn't a prohibition).

The other limitation was inadequate supervision on the part of CHWs and volunteers by their immediate supervisors in specific health facilities. This was affecting some follow-ups to these children after 10 days which in turn, also affected the study. Some CHWs were unable to manage 
their time properly between their day to day work and IPTi follow-up exercise (which was an addition) such that they were failing to visit and collect the diary cards from mothers/caregivers on time.

There was also a limitation of failure to track some registered children due to unidentified relocations especially at Kawale Health centre which is in Lilongwe city. This was very prevalent during month-ends where some women were relocating to other parts of the city without informing $\mathrm{CHWs}$ or volunteers. This made it difficult for $\mathrm{CHWs}$ to know where such children had relocated to.

Misconceptions and myths were some other limitations which made some women stop participating in the study. It was difficult for CHWs and volunteers to convince such parents/caregivers to remain in the study.

There was an incorrect/inaccurate registration of children to be followed at immunisation sites immediately after these children got IPTi. Some CHWs and/or volunteers were unable to correctly list down details of these children's locations which resulted in going to wrong directions during follow-ups in the process missing the right children that needed follow-ups at such particular times.

Although this initiative showed success and that it was recommended to be rolled out, this was eventually not the case. This was so because at that time, SP was the first-line anti-malaria treatment drug which was later changed to Lumefantrine-Artemether (LA) in Malawi before this initiative was implemented. This made it impossible for the initiative to be rolled out as a result; it wasn't implemented at national level.

\section{CONCLUSION}

This study proved to be a success in the sense that it managed to show that SP-IPTi could be combined with immunisation at the same time during childhood EPI vaccinations. It also proved that active surveillance was more effective than passive when following the outcome of a specific drug and/or treatment. The study further showed that there was no Serious Adverse Event following SP-IPTi against malaria in infants. It also showed that it was possible to do this pharmacovigilance at national level and that this approach was a more viable way of assessing if the drug or treatment is compatible with the prevailing conditions in specific targeted groups of people. The study results showed that indeed there was need to combine the anti-malarial drug with childhood immunisation if we were to address the issue of malaria in infants. This approach addressed the accessibility gap parents/caregivers had to health facilities where many children were also protected against malaria besides immunisable diseases.

\section{REFERENCES}

Alexandra de Sousa Leon Paul Rabarijaona Ofori Tenkorang Ebenezer Inkoom Hantamalala V. Ravelomanantena Sabrina Njarasoa Jeremiah Nee Whang Jean Louis Ndiaye Youssoupha Ndiaye Mouhamed Ndiaye (2012). Pharmacovigilance of Malaria Intermittent Preventive Treatment in Infants Coupled With Routine Immunizations in 6 African Countries https://academic.oup.com/jid/article/205/suppl_1/S82/869855 
Coomarasamy A, Williams H, Trucanowicz E. (2016) Definitions of Adverse Events, seriousness and causality. https:www.ccbi.nlm.nih.gov/books/NBK362736/

European Medicines Agency. (2016). Presentation - Pharmacovigilance at European Medicines Agency http://www.ema.europa.eu/docs/en_GB/document_library/Presentation/2016/02/WC500201 046.pdf

Jeetu G, Anusha G. (2010). Pharmacovigilance: A Worldwide Master Key for Drug Safety Monitoring. https://www.ncbi.nlm.nih.gov/pmc/articles/PMC2964775/

Kieran Walshe (2000). Adverse Events in health care: Issues in measurement. Qualitysafety.bmj.com/9/1/47

WHO. (2018) Pharmacovigilance. http://www.who.int/medicines/areas/quality_safety/safety_efficacy/pharmvigi/en/

WHO. (2011) Intermittent preventive treatment for infants using sulfadoxine-pyremethamine (IPTi-SP) for malaria control in Africa: implementation field guide. www.who.int/malaria/publications/atoz/whoivb11_07/en/ 


\section{APPENDICES}

\section{Appendix 1: The diary card}

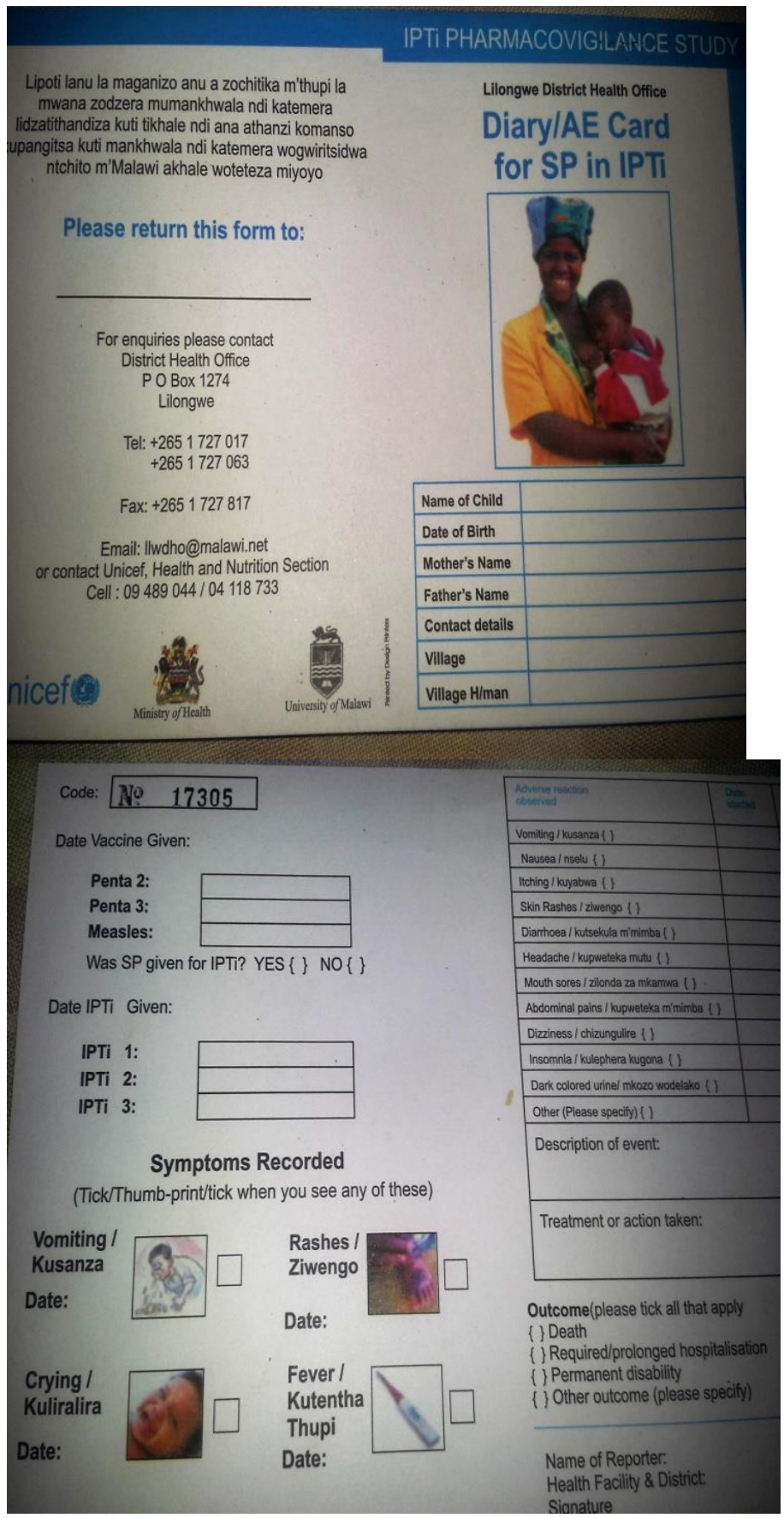




\section{Appendix 2: Database}

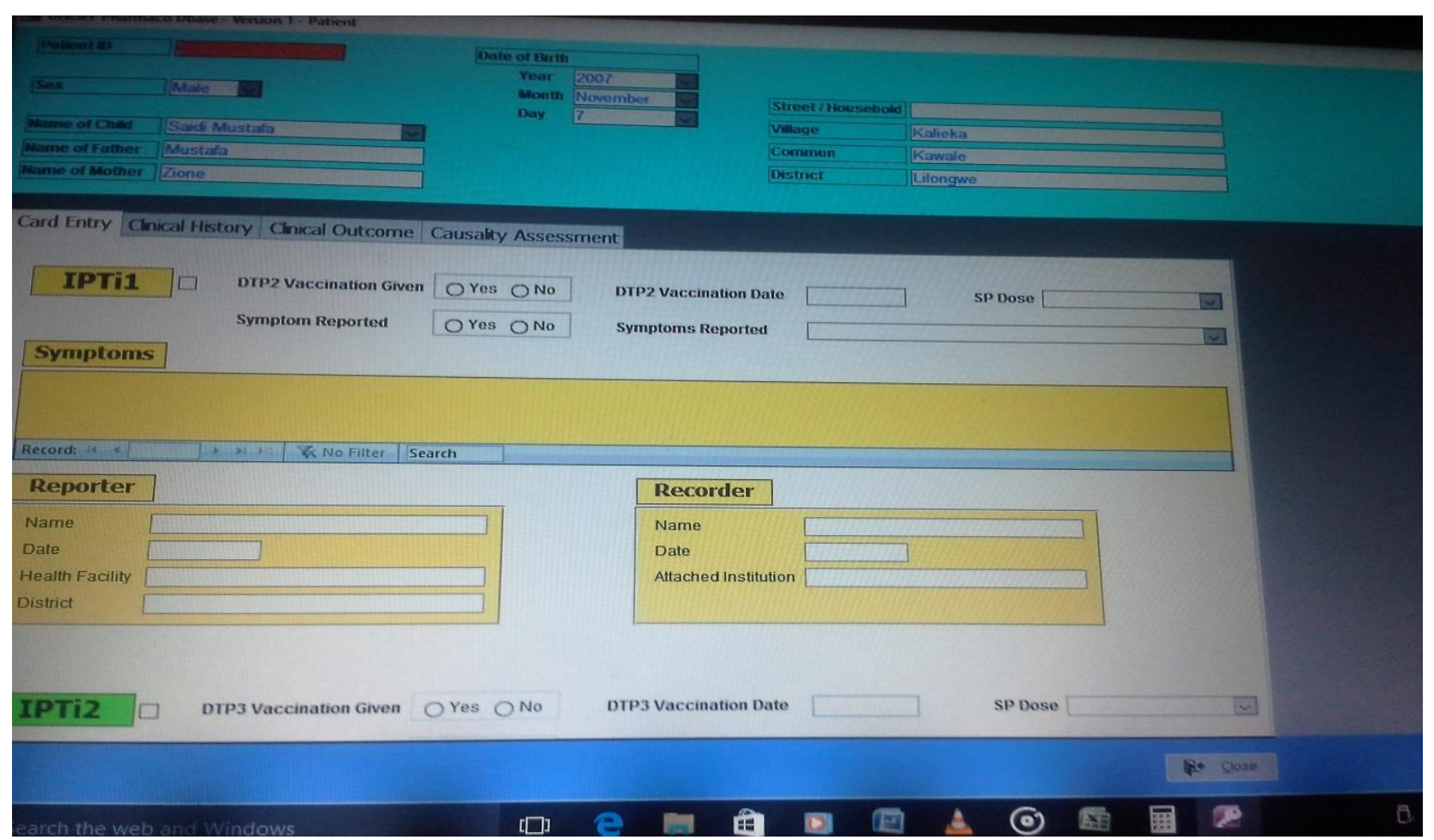

